\title{
Ethnopharmacognosy of Echinops spinosus L. in North Africa: a mini review
}

\author{
Amel Bouzabata ${ }^{1}$, Fawzi Mahomoodally², Carlo Tuberoso ${ }^{3}$ \\ ${ }^{1}$ Faculty of Medicine, Badji-Mokhtar University, Annaba, Algeria \\ ${ }^{2}$ Department of Health Sciences, Faculty of Science, University of Mauritius, Moka, Mauritius \\ ${ }^{3}$ Department of Life and Environmental Sciences, University of Cagliari, Cagliari, Italy
}

\begin{abstract}
Background: The genus Echinops (Asteraceae family, Echinopeae class) consists of ca. 120 species and is native to Africa, the Middle East, Europe, and Asia. In Algeria, this genus is represented by the very common species Echinops spinosus L. also known as "tesskra," which is used as a diuretic, hypoglycemic, for stomachic effects, liver disorders, and post-partum care.

Objective: The aim of this presentation is to provide an overview of the ethnopharmacognosy studies conducted on E. spinosus in North Africa. Data on ethnomedicinal uses, chemical constituents, and pharmacological activity were systematically compiled.

Methods: Several popular search databases, including PubMed, ScienceDirect, Scopus, Web of Science, and Stanford libraries were scrutinised to extract relevant information. The research focused only on English-written papers published between 1980 and 2017. Results: Echinops spinosus L. is traditionally used in North Africa, and it was found that the most ethnomedicinal use reports were from Morocco and Algeria. Promising results have been reported regarding its phytochemistry and pharmacological activity. Fortythree compounds were isolated from different parts of this species. No studies have been conducted to highlight the toxicity and clinical safety of this species.

Conclusion: This review highlights the therapeutic potential of $E$. spinosus used in traditional medicine. Furthermore, clinical trials on standardized preparations are necessary to explore the full safety and efficacy of E. spinosus in North Africa.
\end{abstract}

\section{ARTICLE HISTORY}

Received 18 March 2018

Accepted 30 May 2018

Published 19 June 2018

\section{KEYWORDS}

Echinops species; Algeria; E. spinosus; ethnomedical properties; pharmacology

\section{Introduction}

The genus Echinops, belongs to the family Asteraceae (formerly Compositae) and comprises ca. 120 species distributed throughout the Mediterranean region, in central Asia, and in tropical Africa [1]. In Algeria, this genus is represented by the very common species Echinops spinosus L. According the African Plant Database, as well as the Plant List database, this name is synonymous with E. spinosissimus Turra [2-4]. It thrives in arid desert conditions with an annual rainfall varying between 20 and $100 \mathrm{~mm}$, and has a wide ecological range for soil, including coastal, calcareous dunes, sandy, and gravelly to rocky surfaces [5]. Botanical classifications have subdivided Echinops spinosus L. into two subspecies [6,7]:
E. spinosus ssp. eu. spinosus Maire (var. chaetocephalus Pomel) and E. spinosus ssp. bovei (Boiss.) Maire (var. pallens Maire.), which is also known as $E$. bovei Boiss [8]. Recent data provided by synonymic survey of the Cardueae (Compositae) genera database, validated the scientific name of $E$. spinosissimus subsp. bovei (Boiss.) Greuter $\equiv$ E. bovei Boiss [9].

Three other species have been reported in Algeria, but appear to be not very common: a) $E$. ritro L., known under the name of "oursin bleu" or "echinops" in French, has a southern European distribution, and occurs in southern Europe, western Asia, and even Siberia; b) E. sphaerocephalus L. is a mountainous species; and c) E. strigosus L.

Contact Amel Bouzabata $₫$ amelbouz2009@gmail.com Faculty of Medicine, Badji-Mokhtar University, Annaba, Algeria.

(c) EJManager. This is an open access article licensed under the terms of the Creative Commons Attribution Non-Commercial License (http:// creativecommons.org/licenses/by-nc/3.0/) which permits unrestricted, noncommercial use, distribution and reproduction in any medium, provided the work is properly cited. 
is distributed in the Iberian and North Africa area, especially in southern Spain and Algeria, and is widespread in the most western part of the Tell, from Tenes to the Moroccan border [6]. The literature reveals that 24 species of the genus Echinops have been subjected to varying degrees of scientific investigation [10]. Conversely, very little is known about E. spinosus $[11,12]$. Therefore, the objective of this review is to provide a detailed comparison of the chemical composition and pharmacological properties displayed by E. spinosus in North Africa with the widely studied species. Several search databases, including PubMed, ScienceDirect, Scopus, and Web of Science, were probed to extract information between 1980 and 2017.

\section{Vernacular names}

In Algeria, E. spinosus L. is known in the Berber language under the names "Taskra," "Teskera," "Taskra" Ameskelit T, and Sarsor, and in Arabic by the names: "fouga el djemel," "chouk el djemel," "suk ej-jmal," Kachir, Ikchir, Chouk el Hamir, Chicaou, and Sorr [13-15]. In the Tamahaq language, this species is called Téfariast [16].

The Arabic name of "qounfoudzia" (de hérisson), is the transcription in Greek of "Ekhinos." The vernacular name of "ri ayi el-ibil" has the meaning "Camel Pasture" [14]. In Morocco, this species is known under the names of "tasekra, asekra, teskra, chouk el hamir, suk al-himar, and tîmat" [17].

\section{Botanical description and habitat}

E. spinosus is a perennial herb growing to $1 \mathrm{~m}$ and more, with erect brownish to reddish stems, few long leaves from 10 to $15 \mathrm{~cm}$, hairy, arachnoids, and with very long spines. The inflorescence is often a single hemispherical globe up to $5 \mathrm{~cm}$ in diameter during the flowering period. It is surrounded with numerous long spines (Figure 1). The small hermaphroditic flowers that compose the dense head are tubular, turning from green to white and yellowish when in full bloom. The fruits are small achenes topped by membranous scales to ease dispersion [18].

In Algeria, two very polymorphous subspecies have been described: 1) ssp. bovei (Boiss.) Maire: stems pubescent, not glandular. The achenes are composed into distinct pieces at the base. The leaves are whitish and woolly on both sides. E. bovei is a Southern Mediterranean-Saharan taxon, and is widespread in Algeria [19,20]; and 2) ssp. eu. spinosus Maire: annual plant, upright and firm stems, from 40 to $60 \mathrm{~cm}$. The distribution of $E$. eu spinosus

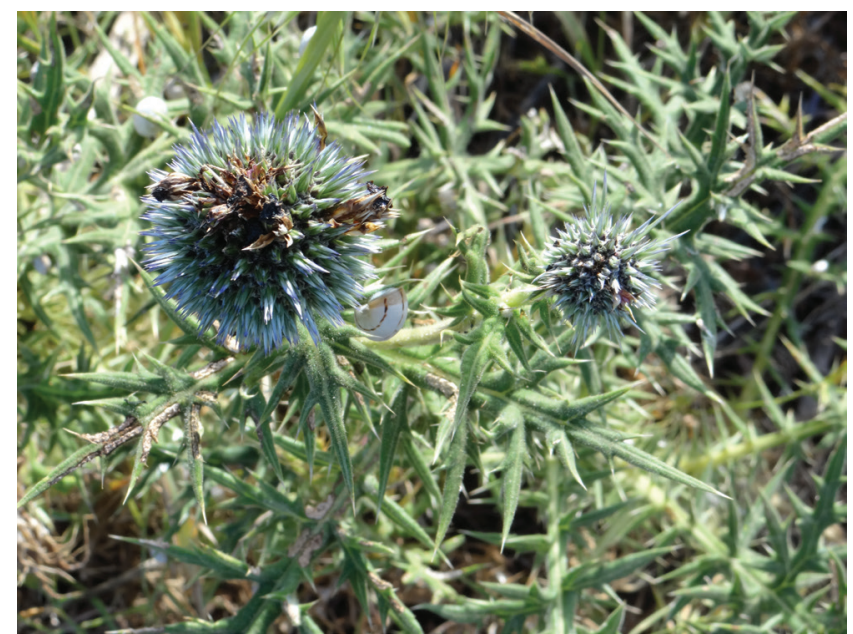

Figure 1. Morphological aspect of Echinops spinosus

is limited to the pre-desert regions in septentrional and central Sahara and is considered as a SaharoSindian taxon $[6,7]$.

\section{Phytochemistry}

The genus Echinops is one of the taxa with characterized alkaloids within the Asteraceae family [21]. An overview of the literature on E. spinosus showed that reports concerning the phytochemistry of the Algerian species are very limited, with only two studies undertaken $[11,12]$. Preliminary qualitative phytochemical screening of various secondary metabolites by specific chemical tests was carried out on extracts of the aerial parts and roots, which indicated that the aqueous extract contained alkaloids, tannins, flavonoids, quinones, reducing sugars, and starch [11].

Phytochemical investigations of E. spinosus from North Africa in Morocco, Algeria, Tunisia, and Egypt led to the isolation and identification of 42 metabolites belonging to the phytochemical classes of quinoline alkaloids $\left(\mathrm{S}_{1}-\mathrm{S}_{2}\right)$ [1], sesquiterpenoids $\left(\mathrm{S}_{3}\right)$ [22], flavonoids $\left(\mathrm{S}_{4}-\mathrm{S}_{26}\right)[12,23]$, and sterols $\left(\mathrm{S}_{27}-\mathrm{S}_{39}\right)[24]$. The names of the isolated compounds and their sources are provided in Table 1 and the chemical structures are depicted in Table 2.

In 2009, the isolation of two sesquiterpenoids with a novel carbon framework was reported and named echinopine A and echinopine B [22]. In 2016, Bouattour et al. [24] identified 13 sterols in E. spinosus from Tunisia. The two most abundant compounds were $ß$-sitosterol (44.97\%) followed by stigmasterol (34.95\%) [24]. In the same year, the occurrence of flavonoids was reported in the aerial parts of E. spinosus from Algeria and Egypt. 
Table 1. Phytochemical constituents of Echinops spinosus.

\begin{tabular}{|c|c|c|c|}
\hline Extract & Compound & Structure & Reference \\
\hline$\overline{E^{a}}$ & $\begin{array}{l}\text { Echinopsine } \\
\text { Echinorine }\end{array}$ & $\begin{array}{l}S_{1} \\
S_{2}\end{array}$ & [1] \\
\hline $\mathrm{E}^{\mathrm{b}}$ & $\begin{array}{l}\text { Echinopine A } \\
\text { Echinopine B }\end{array}$ & $\mathrm{S}_{3}$ & [22] \\
\hline $\mathbf{E}^{\mathrm{c}}$ & $\begin{array}{l}\text { Apigenin } \\
\text { Apigenin-7-O-ß-glucopyranoside } \\
\text { Apigenin-7- } \beta-D-O-\left(6^{\prime \prime}-O-E-p \text {-coumaroyl-glucopyranoside }\right.\end{array}$ & $\begin{array}{l}\mathrm{S}_{4} \\
\mathrm{~S}_{5} \\
\mathrm{~S}_{6}\end{array}$ & [12] \\
\hline $\mathbf{E}^{\mathrm{d}}$ & $\begin{array}{l}\text { Luteolin-6-arabinose-8-glucoside } \\
\text { Luteolin-6-glucose-8-arabinoside } \\
\text { Apigenin-6-arabinose-8-galactoside } \\
\text { Apigenin-6-arabinose-8-glucoside } \\
\text { Apigenin-6-glucose-8-rhamnoside } \\
\text { Luteolin-7-glucoside } \\
\text { Narengin } \\
\text { Rutin } \\
\text { Hesperidin } \\
\text { Quercetin-3-O-glucoside } \\
\text { Rosmarinic acid } \\
\text { Apigenin-7-O-neohespiroside } \\
\text { Kaempferol-3,7-dirhamnoside } \\
\text { Apigenin-7-glucoside } \\
\text { Quercetrin } \\
\text { Quercetin } \\
\text { Naringenin } \\
\text { Hespiritin } \\
\text { Kaempferol } \\
\text { Rhamnetin } \\
\text { Apigenin } \\
\text { Acacetin }\end{array}$ & $\begin{array}{l}S_{7} \\
S_{8} \\
S_{9} \\
S_{10} \\
S_{11} \\
S_{12} \\
S_{13} \\
S_{14} \\
S_{15} \\
S_{16} \\
S_{17} \\
S_{18} \\
S_{19} \\
S_{5} \\
S_{20} \\
S_{21} \\
S_{22} \\
S_{23} \\
S_{24} \\
S_{25} \\
S_{4} \\
S_{26}\end{array}$ & [23] \\
\hline $\mathrm{E}^{\mathrm{e}}$ & $\begin{array}{l}\beta \text {-Sitosterol } \\
\text { Stigmasterol } \\
\text { Campesterol } \\
\text { Brassicasterol } \\
\text { Campestanol } \\
\Delta 7 \text {-Campesterol } \\
\Delta 5,23 \text {-Stigmastadienol } \\
\text { Cholesterol } \\
\text { Sitostanol } \\
\Delta 5 \text {-Avenasterol } \\
\Delta 5,24-\text { Stigmastadienol } \\
\Delta 7 \text {-Stigmastenol } \\
\Delta 7 \text {-Avenasterol }\end{array}$ & $\begin{array}{l}S_{27}^{26} \\
S_{28} \\
S_{29} \\
S_{30} \\
S_{31} \\
S_{32} \\
S_{33} \\
S_{34} \\
S_{35} \\
S_{36} \\
S_{37} \\
S_{38} \\
S_{39}\end{array}$ & [24] \\
\hline $\mathbf{E}^{\mathrm{f}}$ & 2,2-Ddimethyl-4 [5'-(prop-1-ynyl)-2,2'-biothiphen-5-yl]-1,3-dioxalane & $\mathbf{S}_{40}^{39}$ & [25] \\
\hline $\mathrm{E}^{\mathrm{g}}$ & 11-Hydroxyisocom-2-en-5-one & $\mathrm{S}_{41}$ & {$[26]$} \\
\hline $\mathrm{E}^{\mathrm{h}}$ & A-neooleana-3(5),12-diene & $S_{42}$ & [27] \\
\hline
\end{tabular}

$E^{a}=$ chloroform extract prepared from ripe fruits collected in Deltaic coast Egypt, $E^{b}=$ methanol extract prepared from root collected in Morocco (2003), $\mathrm{E}^{\mathrm{c}}=$ ethyl acetate extract prepared from aerial parts collected in North Eastern Algeria (April 2009), $\mathrm{E}^{\mathrm{d}}=$ aqueous ethanolic extract prepared from aerial parts collected in Egypt, $\mathrm{E}^{\mathrm{e}}=$ Hexane extract prepared from flower heads in Tunisia, $E^{f}=$ dichloromethane extract prepared from roots collected in Morocco, $\mathrm{E}^{\mathrm{g}}=$ dichloromethane extract prepared from roots collected in Morocco, $\mathrm{E}^{\mathrm{h}}=$ crude methanol and ethyl acetate extracts from flowers collected from Sfax, South Tunisia (June 2011).

Twenty-three flavonoids were isolated [12,23]. One year later, Bouattour et al., isolated a new derivative of apigenin named apigenin-7-O-ß-D-glucoside(4"-O-trans-p-coumaroyl) [27].

The occurrence of simple quinoline alkaloids in the aerial and/or underground parts was reported in E. ritro, E. echinatus Roxb [28,29], E. albicaulis
[30], and E. niveus [31]. Furthermore, members of the genus Echinops are also reported to contain flavonoids, triterpenoids, and thiophene acetylenes [21,29,30,32-34].

As far as information provided in the literature, thiophenes are a class of heterocyclic compounds which are characteristic secondary 
Table 2. Chemical structures of isolated compound extracted from Echinops spinosus L.<smiles>Cn1ccc(=O)c2ccccc21</smiles>

$\mathrm{S}_{1}$

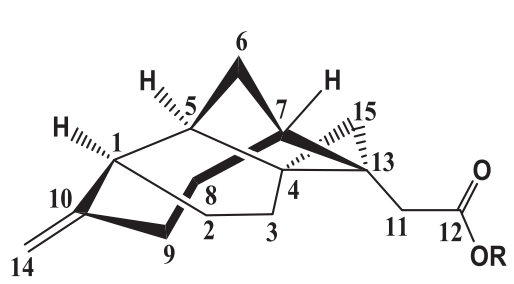

(A)

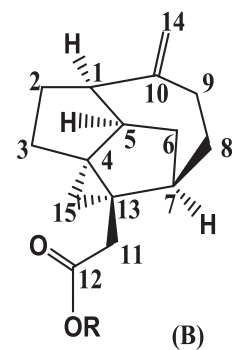

(B)

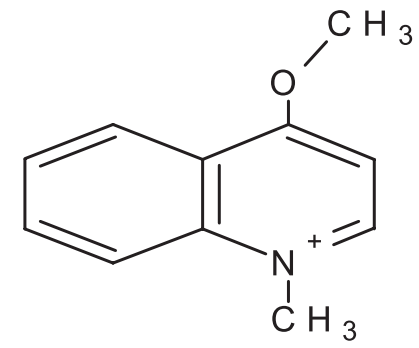

$\mathrm{S}_{2}$<smiles>O=c1cc(-c2ccc(O)cc2)oc2cc(O)cc(O)c12</smiles>

(A) $\mathrm{R}=\mathrm{H}$
(B) $\mathrm{R}=\mathrm{CH}_{3}$

$\mathrm{S}_{3}$<smiles>O=c1cc(-c2ccc(O)cc2)oc2cc(O[C@@H]3O[C@H](CO)[C@@H](O)[C@H](O)[C@H]3O)cc(O)c12</smiles>

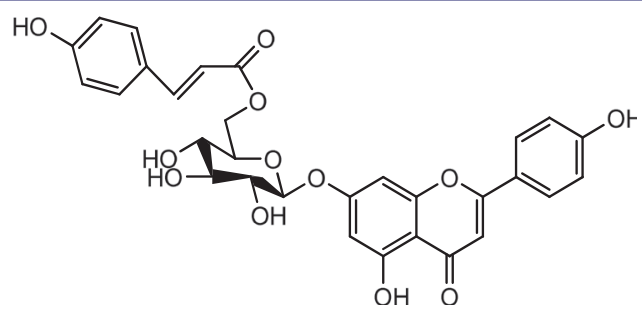<smiles>O=c1cc(-c2ccc(O)c(O)c2)oc2c(C3OCC(O)C(O)C(O)C3O)c(O)c(C3OCC(O)C(O)C3O)c(O)c12</smiles>

$S_{7}$

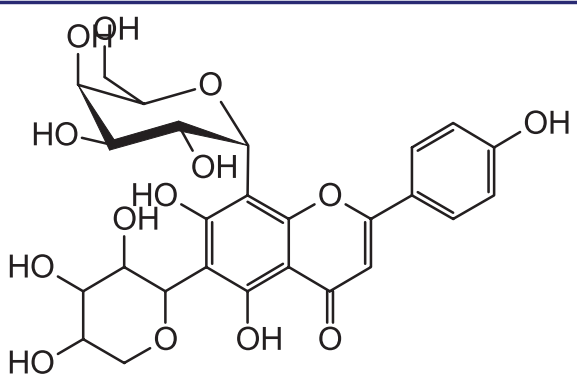<smiles>O=c1cc(-c2ccc(O)cc2)oc2c(C3OCC(O)C(O)C(O)C3O)c(O)c(C3OCC(O)C(O)C3O)c(O)c12</smiles>

$\mathrm{S}_{9}$

$\mathbf{S}_{10}$ 
Table 2. Chemical structures of isolated compound extracted from Echinops spinosus L. (Continued)

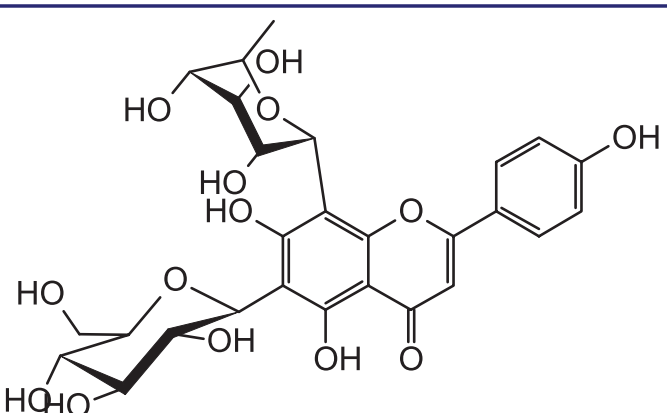<smiles>O=c1cc(-c2ccc(O)c(O)c2)oc2cc(OC(O)[C@H](O)[C@H](O)CO)cc(O)c12</smiles>

$\mathrm{HQHO}$

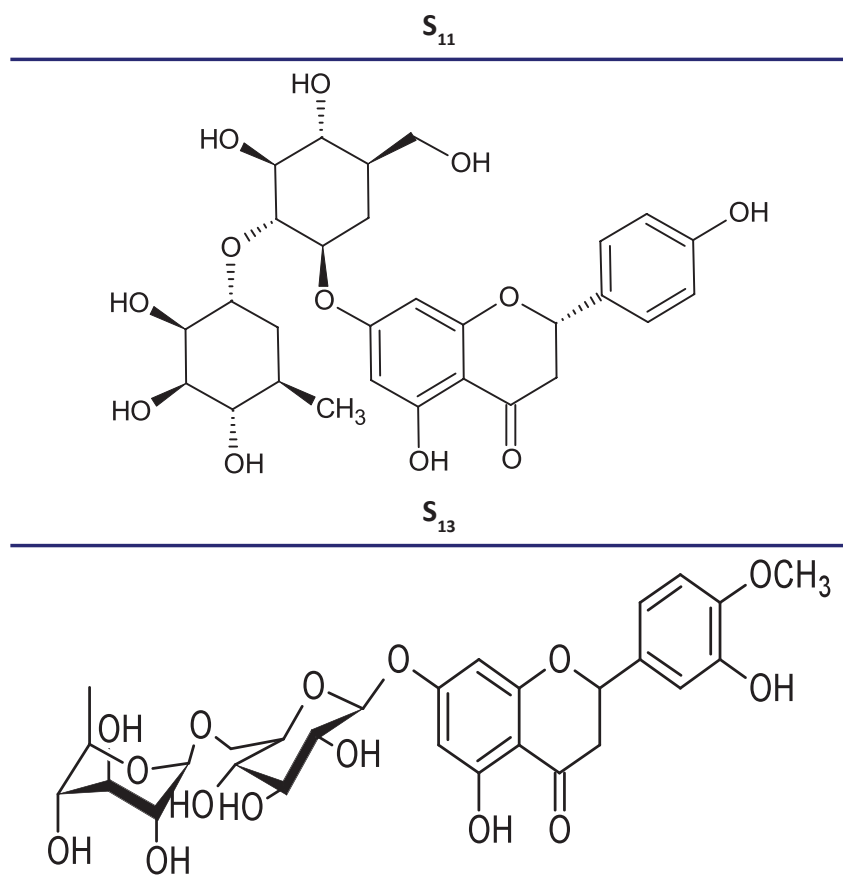

$\mathrm{S}_{12}$

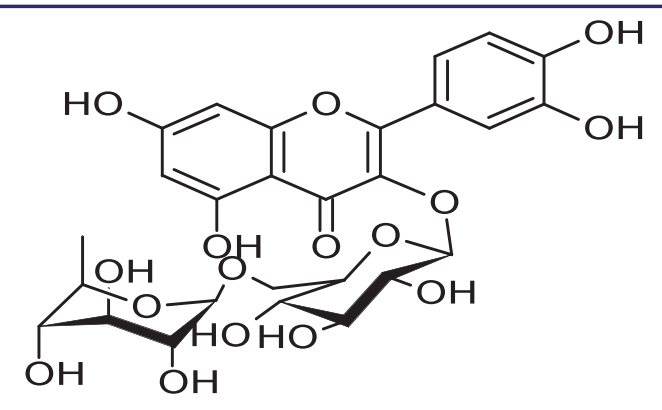

$$
\mathrm{S}_{14}
$$<smiles>O=c1c(O)c(-c2ccc(O)c(O)c2)oc2cc(O)cc(O)c12</smiles><smiles>OC1OC2OC1C(O)C(O)C(O)C(O)C2O</smiles><smiles>C[C@@H]1O[C@H](O[C@@H]2[C@@H](Oc3cc(O)c4c(=O)cc(-c5ccc(O)cc5)oc4c3)O[C@H](CO)[C@@H](O)[C@H]2O)[C@H](O)[C@@H](O)[C@H](O)[C@@H]1O</smiles><smiles>C[C@@H]1O[C@H](Oc2cc(O)c3c(=O)c(O[C@@H]4O[C@H](C)[C@@H](O)[C@H](O)[C@H]4O)c(-c4ccc(O)cc4)oc3c2)[C@H](O)[C@H](O)[C@@H]1O</smiles>

$\mathbf{S}_{19}$ 
Table 2. Chemical structures of isolated compound extracted from Echinops spinosus L. (Continued)<smiles>C[C@@H]1O[C@H](Oc2c(-c3ccc(O)c(O)c3)oc3cc(O)cc(O)c3c2=O)[C@H](O)[C@H](O)[C@@H]1O</smiles><smiles>O=c1c(O)c(-c2ccc(O)c(O)c2)oc2cc(O)cc(O)c12</smiles><smiles>O=C1CC(c2ccc(O)cc2)Oc2cc(O)cc(O)c21</smiles>

$\mathbf{S}_{21}$<smiles>COc1ccc([C@H]2CC(=O)c3c(O)cc(O)cc3O2)cc1O</smiles>

$\mathrm{S}_{22}$<smiles>COc1ccc(-c2cc(=O)c3c(O)cc(O)cc3o2)cc1OC</smiles><smiles>COc1cc(O)c2c(=O)c(O)c(-c3ccc(O)c(O)c3)oc2c1</smiles>

$\mathrm{S}_{24}$

$\mathbf{S}_{25}$<smiles>COc1ccc(-c2cc(=O)c3c(O)cc(O)cc3o2)cc1</smiles>

$\mathbf{S}_{26}$

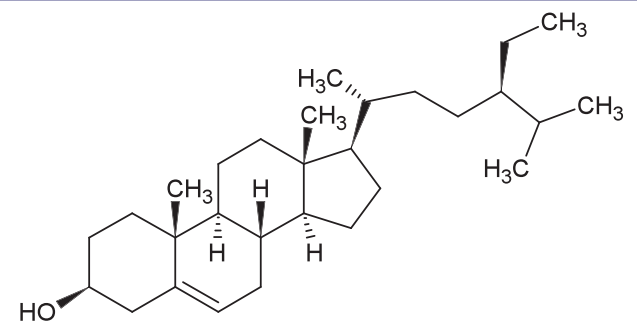

$\mathrm{S}_{27}$<smiles>CC[C@H](/C=C/[C@@H](C)[C@H]1CC[C@H]2[C@@H]3CC=C4C[C@@H](O)CC[C@]4(C)[C@H]3CC[C@@]21C)C(C)C</smiles>

$\mathrm{S}_{28}$ 
Table 2. Chemical structures of isolated compound extracted from Echinops spinosus L. (Continued)
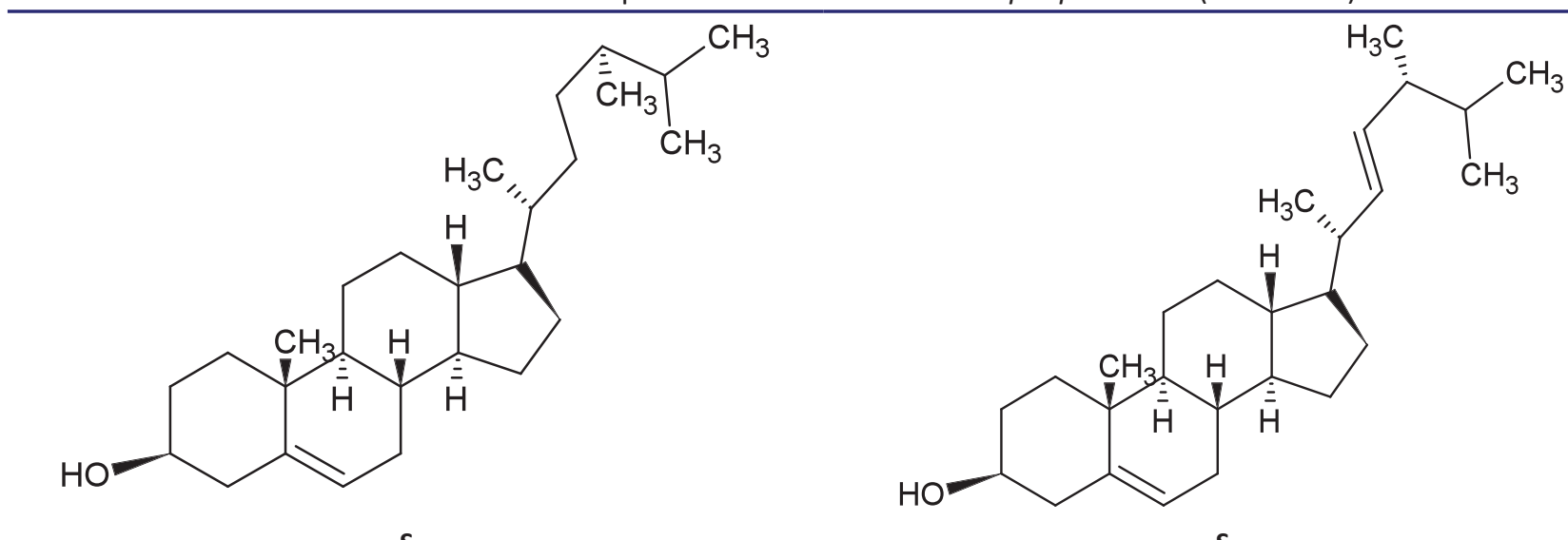

$\mathbf{S}_{29}$

$\mathrm{S}_{30}$<smiles>CC(C)[C@@H](C)CC[C@H](C)[C@H]1CCC2[C@@H]1CC[C@H]1[C@H]2CC[C@H]2C[C@@H](O)CC[C@H]21</smiles><smiles>CC(C)[C@H](C)CC[C@@H](C)C1CCC2C3=CCC4C[C@@H](O)CC[C@]4(C)C3CC[C@]21C</smiles><smiles>CCC(=CC[C@@H](C)C1CCC2C3CC=C4C[C@@H](O)CC[C@]4(C)C3CC[C@@]21C)C(C)C</smiles>

$\mathrm{S}_{32}$

$\mathbf{S}_{33}$
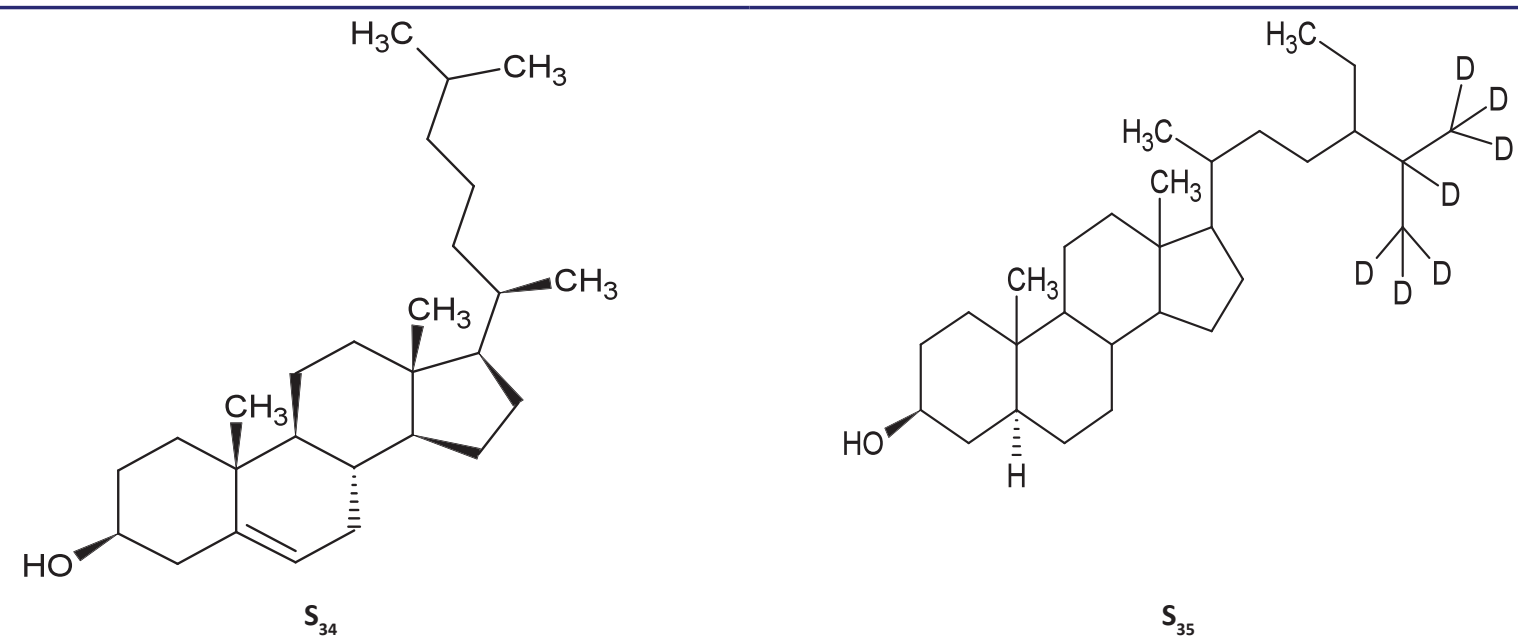
Table 2. Chemical structures of isolated compound extracted from Echinops spinosus L. (Continued)

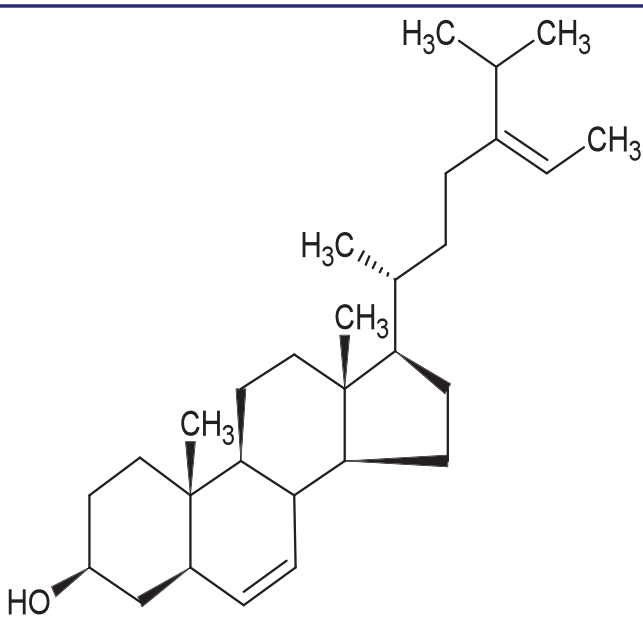

$\mathrm{S}_{36}$
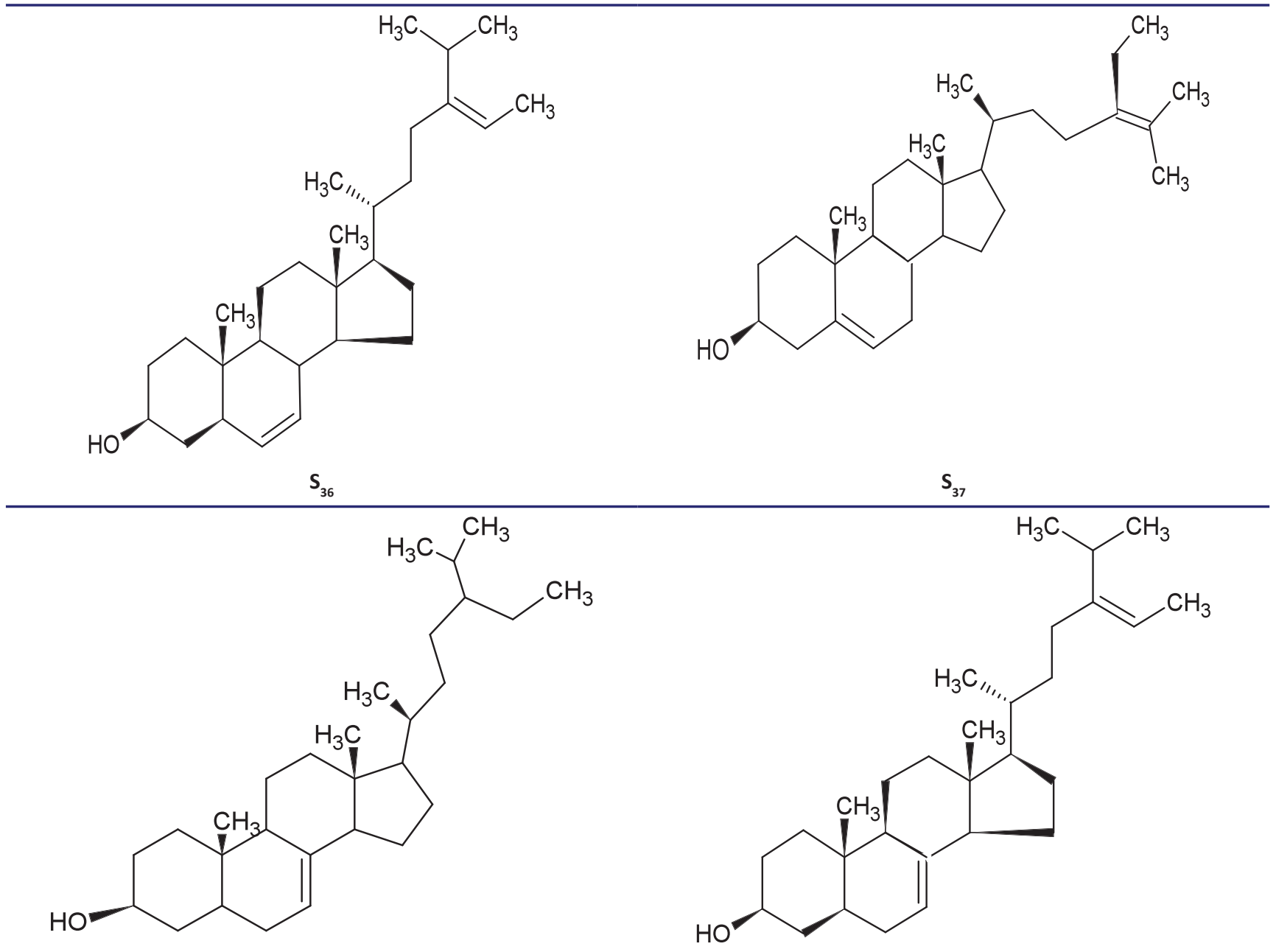

$\mathbf{S}_{37}$

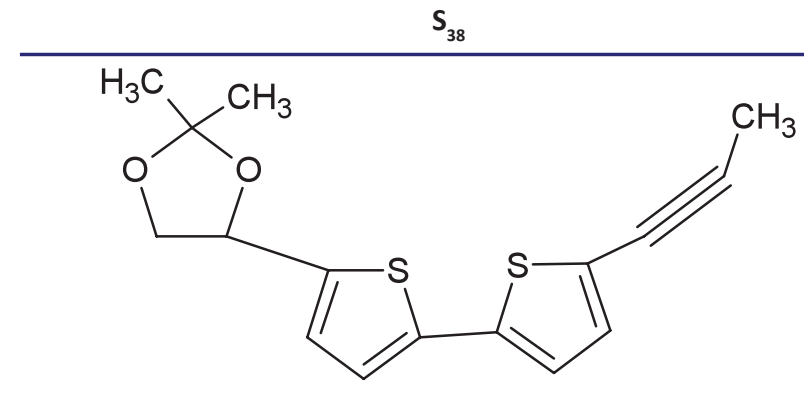

$\mathrm{S}_{39}$

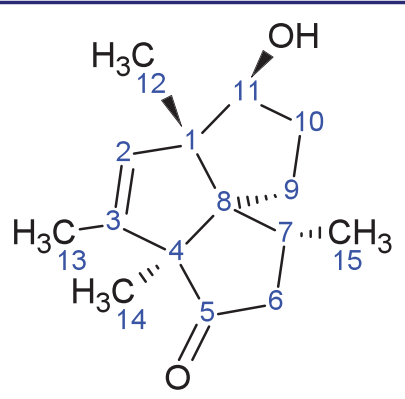

$\mathbf{S}_{40}$

$\mathrm{S}_{41}$<smiles>CC(C)C1=C2CC[C@H](C)[C@@]3(C)CCC(=CC[C@@]23C)[C@@H]2CC(C)(C)CC[C@]12C</smiles>

$\mathrm{S}_{42}$ 
Table 3. Traditional uses and ethnobotanical information on Echinops spinosus.

\begin{tabular}{|c|c|c|c|c|c|}
\hline Country & Plant part & Popular name & $\begin{array}{l}\text { Geographical } \\
\text { distribution }\end{array}$ & Medicinal uses & Reference \\
\hline Morocco & Root & Tassekra & Spontaneous & $\begin{array}{l}\text { Diuretic, hypoglycemic, stomachic, for } \\
\text { liver disorders, and post-partum care }\end{array}$ & [17] \\
\hline Morocco & Root & $\begin{array}{l}\text { Tassekra, Kherchouf, } \\
\text { Chouk al-Himar }\end{array}$ & Not indicated & $\begin{array}{l}\text { Hypoglycemic, diuretic, post-partum } \\
\text { care, liver disorders, appetizing, } \\
\text { depurative, and abortive }\end{array}$ & [38] \\
\hline Morocco & Not indicated & Not indicated & Not indicated & $\begin{array}{l}\text { Against cold, and pain, renal colic, and } \\
\text { disinfectant. Warming plant }\end{array}$ & [39] \\
\hline Morocco & Root & Tassekra & Spontaneous & $\begin{array}{l}\text { Obstetric } \\
\text { Decoction of roots is administered } \\
\text { to women after delivery to expel the } \\
\text { placenta and for blood flow }\end{array}$ & [40] \\
\hline Morocco & $\begin{array}{l}\text { Root and aerial } \\
\text { parts } \\
\text { Roots and branches } \\
\text { Flowers }\end{array}$ & Taskra, Bongar & Hemicryptophyte & $\begin{array}{l}\text { Decoction used for colds, kidney, } \\
\text { stones disinfectant, diuretic, and } \\
\text { hypoglycemic. } \\
\text { Decoction: abortifacient, labor pains } \\
\text { Tisane: neuralgia, fatigue }\end{array}$ & [41] \\
\hline Morocco & Seeds & Taskra & Not indicated & Infusion is used as an antidiabetic & {$[42]$} \\
\hline Morocco & Rhizomes & Taskra & Not indicated & Decoction: stomachic disorders & [43] \\
\hline $\begin{array}{l}\text { Algeria } \\
\text { Mascara }\end{array}$ & Roots & Tassekra & Not indicated & $\begin{array}{l}\text { Genital infections (after an abortion), } \\
\text { urinary tract infections, inflammation } \\
\text { of the kidneys, and blood circulation }\end{array}$ & [44] \\
\hline AlgeriaTiaret & Not indicated & Taskra & Not indicated & $\begin{array}{l}\text { Hygienic agent employed for } \\
\text { gynecological reasons }\end{array}$ & [45] \\
\hline AlgeriaSetif & Roots and Fruits & Not indicated & $\begin{array}{l}\text { Therophyte, southern } \\
\text { Mediterranean Sahara }\end{array}$ & Labor pains, abortion, and neuralgia & [19] \\
\hline $\begin{array}{l}\text { AlgeriaTassili des } \\
\text { N'jjers }\end{array}$ & Aerial Part & $\begin{array}{l}\text { Tefaryast } \\
\text { Tassegra }\end{array}$ & Saharo-arabic & $\begin{array}{l}\text { Infusion in internal use: } \\
\text { Eye complaints, trachoma, sore } \\
\text { inflammation, digestive diseases, } \\
\text { spasms, colic, and fever }\end{array}$ & [20] \\
\hline
\end{tabular}

metabolites derived from plants belonging to the family Asteraceae such as Echinops. Based on this, the distribution of thiophenes in different species of the genus Echinops has been examined in six species, including: E. grijissii Hance, E. pappii Chiov, E. hispidus Fresen, E. transiliensis Golosh, E. latifolius Taush, and E. ritro L. [34]. Twenty thiophenes have been reported in nine Ethiopian species: $E$. amplexicaulis, E. papii, E. ellenbeckii, E. hispidus, E. hoehnelii, E.kebericho, E. longisetus, E. macrochaetus, and E. giganteus [10]. In parallel, it should be noted that E. spinosus has not been screened for thiophene composition; only one chemical report described the structure of a new thiophene in roots collected from Morocco, which is known as the acetylene 2,2-dimethyl-4 [5'-(prop-1-ynyl)-2,2'-bithiophen-5-yl]-1,3-dioxalane $\left(\mathrm{S}_{40}\right)$ [26]. Furthermore, a new sesquiterpenoid 11-hydroxyisocom-2-en5-one $\left(\mathrm{S}_{41}\right)$ was described for the first time in the dichloromethane extract of the roots of E. spinosissimus subsp. spinosus Greuter from Morocco [22]. In 2017, Bouattour et al., isolated a $\mathrm{C}_{30}$-pentacyclic triterpadiene A-neooleana-3(5),12-diene $\left(\mathrm{S}_{42}\right)$, which might be a marker of identification of E. spinosus from other species of Echinops [27].

\section{Ethnobotanical aspects}

Traditional preparations of E. spinosus are frequently used in folk medicine as an abortifacient, as a diuretic, and for blood circulation, diabetes, gastric pain, indigestion, and spasmolytic problems [35].

In traditional medicine practices, E. spinosus is known in the Chinese [36] and North African traditions [37]; the latter reporting the ethnomedicinal use of the stems, leaves, and roots as a diuretic drug.

In Algeria, the roots or flower heads of E. spinosus have been used in the treatment of prostatism and dysmenorrhea. This botanical remedy has also been used as a peripheral vasoconstrictor in the treatment of hemorrhoids, varicose veins, and varicocele, in various venous hemorrhages and in metrorrhagia. It is considered as a hypertensive drug $[13,14]$. Table 3 presents the diverse ethnomedicinal uses of various parts of $E$. spinosus in North Africa. 
Table 4. In vivo anti-inflammatory properties of E. spinosus and E. echinatus.

\begin{tabular}{|c|c|c|c|c|c|c|c|}
\hline Species & $\begin{array}{l}\text { Extract, Plant } \\
\text { Part }\end{array}$ & $\begin{array}{c}\text { Dose: } \\
\text { dmi, dma }\end{array}$ & Inhibition & Inflammation model & $\begin{array}{c}\text { Route of } \\
\text { administration }\end{array}$ & $\begin{array}{l}\text { Control- } \\
\text { dose \% of } \\
\text { inhibition }\end{array}$ & Reference \\
\hline \multirow[t]{3}{*}{ E. spinosus } & Aqueous, $\mathrm{RH}$ & $100 \mathrm{mg} / \mathrm{kg}$ & $59.5 \%^{*}$ & $\begin{array}{l}\text { Carrageenan-induced } \\
\text { rat sub-plantar edema }\end{array}$ & i.p. & IMC3 mg/kg & [47] \\
\hline & Ethanol, RH & $100 \mathrm{mg} / \mathrm{kg}$ & $21.3 \%^{*}$ & & & & \\
\hline & Chloroform, RH & $100 \mathrm{mg} / \mathrm{kg}$ & $67.4 \%^{*}$ & & & & \\
\hline \multirow[t]{3}{*}{ E. spinosus } & Aqueous, $\mathrm{RH}$ & $3 \mathrm{mg} / \mathrm{ear}$ & $\mathrm{NI}$ & $\begin{array}{l}\text { Arachidonic acid- } \\
\text { induced mouse ear } \\
\text { edema }\end{array}$ & i.p. & IMC1 mg/kg & [47] \\
\hline & Ethanol, $\mathrm{RH}$ & $3 \mathrm{mg} / \mathrm{ear}$ & $51.0 \%$ & & & & \\
\hline & Chloroform, RH & $3 \mathrm{mg} / \mathrm{ear}$ & $56.0 \%$ & & & & \\
\hline \multirow[t]{2}{*}{ E. echinatus } & Ethanol, WP & $100 \mathrm{mg} / \mathrm{kg}$ & $\begin{array}{l}\text { i.p.: } 38.9 \%^{* *} \\
\text { i.o.: } 13.3 \%^{* *}\end{array}$ & $\begin{array}{l}\text { Carrageenan-induced } \\
\text { rat sub-plantar edema }\end{array}$ & $\begin{array}{l}\text { i.p. } \\
\text { i.o. }\end{array}$ & PBZ5 mg/kg & [51] \\
\hline & & $800 \mathrm{mg} / \mathrm{kg}$ & $\begin{array}{l}\text { i.p.: } 67.5 \%^{* *} \\
\text { i.o.: } 51.8 \%^{* *}\end{array}$ & & & & \\
\hline \multirow[t]{2}{*}{ E. echinatus } & Ethanol, WP & $25 \mathrm{mg} / \mathrm{kg}$ & $\begin{array}{l}\text { a.c: } 18.2 \%^{* * *} \\
\text { a.ch: } 32.3 \%^{* *}\end{array}$ & $\begin{array}{l}\text { Formaldehyde-induced } \\
\text { acute and chronic } \\
\text { reactions in rats }\end{array}$ & $\begin{array}{l}\text { a.c: }+4 \text { hours } \\
\text { reaction. } \\
\text { a.ch: }+10 \text { days }\end{array}$ & PBZ50 mg/kg & [51] \\
\hline & & $200 \mathrm{mg} / \mathrm{kg}$ & $\begin{array}{l}\text { a.c: } 50.3 \%^{* * *} \\
\text { a.ch: } 54.3 \%{ }^{* *}\end{array}$ & & & & \\
\hline \multirow[t]{2}{*}{ E. echinatus } & Ethanol, WP & $25 \mathrm{mg} / \mathrm{kg}$ & $\begin{array}{l}\text { a.c: } 23.6 \%^{* *} \\
\text { a.ch: } 44.1 \%^{* *}\end{array}$ & $\begin{array}{l}\text { Adjuvant-induced acute } \\
\text { and chronic reactions } \\
\text { in rats }\end{array}$ & $\begin{array}{l}\text { a.c: }+18 \text { hours } \\
\text { reaction. } \\
\text { a.ch: }+21 \text { days }\end{array}$ & PBZ50 mg/kg & [51] \\
\hline & & $200 \mathrm{mg} / \mathrm{kg}$ & $\begin{array}{l}\text { a.c: } 75.4 \%^{* *} \\
\text { a.ch: } 75.6 \%{ }^{* *}\end{array}$ & & & & \\
\hline \multirow[t]{2}{*}{ E. echinatus } & TA & $12.5 \mathrm{mg} / \mathrm{kg}$ & $13.79 \%^{* *}$ & $\begin{array}{l}\text { Carrageenan-induced } \\
\text { rat sub-plantar edema }\end{array}$ & & $\begin{array}{l}\mathrm{PBZ} 50 \mathrm{mg} / \mathrm{kg} \\
46.23 \%{ }^{* *}\end{array}$ & [52] \\
\hline & & $200 \mathrm{mg} / \mathrm{kg}$ & $63.25 \%{ }^{* *}$ & & & & \\
\hline \multirow[t]{2}{*}{ E. echinatus } & TA & 10 mg/kg & $\begin{array}{l}\text { a.c: } 17.72 \%^{* * *} \\
\text { a.ch: } 29.70 \%^{* *}\end{array}$ & $\begin{array}{l}\text { Formaldehyde-induced } \\
\text { inflammation }\end{array}$ & $\begin{array}{l}\text { a.c: }+4 \text { hours } \\
\text { reaction. } \\
\text { a.ch: }+10 \text { days }\end{array}$ & $\begin{array}{l}\text { PBZ50 mg/kg } \\
\text { a.c: } 22.69 \%{ }^{* *} \\
\text { a.ch: } 56.15 \%^{* *}\end{array}$ & {$[52]$} \\
\hline & & $80 \mathrm{mg} / \mathrm{kg}$ & $\begin{array}{l}\text { a.c: } 50.70 \%^{* *} \\
\text { a.ch: } 70.64 \%^{* *}\end{array}$ & & & & \\
\hline \multirow[t]{2}{*}{ E. echinatus } & TA & $10 \mathrm{mg} / \mathrm{kg}$ & $\begin{array}{l}\text { a.c: } 29.50 \%{ }^{* *} \\
\text { a.ch: } 39.62 \%^{* *}\end{array}$ & $\begin{array}{l}\text { Adjuvant-induced } \\
\text { inflammation }\end{array}$ & $\begin{array}{l}\text { a.c: }+18 \text { hours } \\
\text { reaction. } \\
\text { a.ch: }+21 \text { days }\end{array}$ & $\begin{array}{l}\text { PBZ50 mg/kg } \\
\text { a.c: } 34.16 \%{ }^{* *} \\
\text { a.ch: } 64.21 \%^{* *}\end{array}$ & [52] \\
\hline & & $80 \mathrm{mg} / \mathrm{kg}$ & $\begin{array}{l}\text { a.c: } 57.91 \%^{* *} \\
\text { a.ch: } 67.47 \%^{* *}\end{array}$ & & & & \\
\hline E. echinatus & Flavanone A & $45 \mathrm{mg} / \mathrm{kg}$ & $34.21 \%$ & $\begin{array}{l}\text { Carrageenan-induced } \\
\text { paw oedema }\end{array}$ & i.p. & $\begin{array}{c}\text { ASA30 mg/kg } \\
52.63 \%\end{array}$ & [53] \\
\hline
\end{tabular}

$\mathrm{dmi}=$ minimal dose, $\mathrm{dma}=$ maximal dose, $\mathrm{RH}=$ rhizome, $\mathrm{WP}=$ whole plant, $\mathrm{NI}=$ not investigated, i.p. $=$ intraperitoneal administration, i.o. $=$ oral administration, a.c. $=$ acute reaction, a.ch. $=$ chronic reaction,

TA = taraxasterol acetate, Flavanone $A=5,7$-dihydroxy-8,4'-dimethoxy-flavanone-5-O- $\alpha$-L-rhamnopyranosyl-7-O- $\beta$-D-arabinopyranosyl$(1 \rightarrow 4)-O-\beta-D$-glucopyranoside, IMC = indomethacin, ASA = acetyl salicylic acid.

$* p<0.02, * * p<0.01, * * * p<0.05$.

\section{Pharmacological properties}

\section{Anti-inflammatory activity}

Over a long period of time, many medicinal plants have been used for the treatment and management of various forms of inflammatory conditions by African traditional healers and herbalists. However, most of these plants are not documented as compared to the Chinese or Indian traditional medicines. About 5,000 plant species have used for centuries for the treatment of various diseases, including anti-inflammatory diseases. A few African medicinal plants with demonstrated anti-inflammatory and analgesic properties have been documented in the last two decades [18].

The genus Echinops is used traditionally in North Africa for its anti-inflammatory actions [46]. In 1999, Rimbau et al., assessed the anti-inflammatory activities of the aqueous, ethanol, and chloroform extracts from the rhizome of E. spinosus [47]. Two experimental methods were used: a) carrageenan-induced rats sub-plantar edema inflammatory 
models $[48,49]$ with administered extracts at a dose of $100 \mathrm{mg} / \mathrm{kg}$ and the reference group was treated with indomethacin (intraperitoneal administration: i.p., $3 \mathrm{mg} / \mathrm{kg}$ ), and b) the arachidonic acid-induced mouse ear edema $[49,50]$, where extracts were studied at a dose of $3 \mathrm{mg} / \mathrm{ear}$, and the reference group was treated with indomethacin (1 mg/ear). All of the tested extracts showed significant anti-inflammatory activities in both experimental models. However, the chloroform extract showed higher anti-inflammatory activity for the carrageenan experimental model inhibition in rats, with a mean of percentage inhibition of $67.4 \%$, compared with $32.4 \%$ for the reference group. In the experimental model in mice, the percentage inhibition for the chloroform extract was $56.1 \%$, compared with $34 \%$ for the reference group. Table 4 summarizes the model of inflammation, the extracts used in the study, and the positive control used.

In parallel, a wide range of anti-inflammatory activity has been shown for E. echinatus used in the Indian System of Medicine for the treatment of fever and inflammatory diseases. In 1989, E. echinatus L. was extensively studied for its acute anti-inflammatory induced in rats by carrageenan, formaldehyde-induced acute and chronic arthritis, and adjuvant-induced acute and chronic arthritis. Taking in to account the methods described to assess the anti-inflammatory activity of E. spinosus [48], it was found that the ethanol extract of the whole plant of E. echinatus at a dose of $100 \mathrm{mg} / \mathrm{kg}$ was less effective than E. spinosus. It is noticeable that the percentage of inhibition in the acute carrageenan paw edema was higher in intraperitoneal (i.p.) than oral (p.o.) dosing, with a percentage of inhibition 38.9\% $\pm 2.5 \%$, versus $13.3 \% \pm 5.3 \%$, compared with the reference group treated with Phenylbutazone (PBZ) (5 $\mathrm{mg} / \mathrm{kg}$ ) of $61.3 \% \pm 2.8 \%$ (i.p.), versus $44.6 \% \pm 4.3 \%$ for i.o. administration [51].

Another study isolated a triterpenoid taraxasterol acetate from E. echinatus and was reported to have an anti-inflammatory effect in albino rats for carrageenan, formaldehyde, and adjuvant-induced inflammation. The effects were dose dependent, and its efficacy was approximately $0.25-2$ times that of the reference drug PBZ administrated per i.p. [52].

Interestingly, a new anti-inflammatory agent has been isolated from the methanolic extract from leaves of $E$. echinatus, 5,7-dihydroxy-8,4'-dimethoxy-flavanone-5- $O$ - $\alpha$-L-rhamnopyranosyl-7- $O-\beta$-D-arabinopyranosyl-( $1 \longrightarrow 4)-O$ - $\beta$-D-glucopyranoside carried out with non-immunological carragenan-induced hind paw oedema method, which showed an in i.p. administration an inhibitory effect 0.67 times that of the reference drug (Table 4) [53].

\section{Antioxidant activity}

The antioxidant activity of flavonoids and tannin extracts from the aerial part (stems and leaves) and roots of E. spinosus from Tlemcen was assessed by two methods: the reduction of ferric reducing antioxidant power (FRAP) and the trapping of free radical 2,2-diphenyl-1-picrylhydrazyl (DPPH). This study showed that the tannin-containing ethyl acetate extract of the aerial arts of E. spinosus had higher capacity of reducing iron and also free radical scavenging activity in the DPPH test than extracts of the roots. The inhibitory concentration $\left(\mathrm{IC}_{50}\right)$ in the DPPH method was $8.25 \mu \mathrm{g} / \mathrm{ml}$ for the aerial parts (vs. $23 \mu \mathrm{g} / \mathrm{ml}$ for roots), while the FRAP method confirmed the high reduction capacity at a concentration of $2.5 \mathrm{mg} / \mathrm{ml}$ (optical density = 2.85) of tannin-containing ethyl acetate extract of aerial parts compared with the root extracts [11]. Similarly, Khedher et al. (2014) showed that the ethanol extract of E. spinosus had the greatest ability to reduce DPPH radicals, with an $\mathrm{IC}_{50}$ value of $147 \mu \mathrm{g} / \mathrm{ml}$. As expected, it was reported for the roots of E. spinosus that there is a positive correlation between the condensed tannin content and activity in the DPPH assay [35].

Comparatively, high scavenging DPPH activities were shown for the methanolic extract of seeds and leaves of E. orientalis [54]. It was shown that the aqueous extract of $E$. ritro is a source of phenolic compounds based on gallic acid, measured by FolinCiocalteau methods (92.24 Gallic Acid Equivalents (GAE) mg/100 g), and exhibited higher DPPH scavenging activity compared with a synthetic antioxidant Butylated hydroxytoluene (BHT) [55].

\section{Antimicrobial activity}

The antibacterial and the antifungal activities of the unsaponifiable matter, and a fraction isolated from the hexane extract of E. spinosus, were evaluated for their antimicrobial potential against eight Grampositive and Gram-negative bacteria by measuring the diameter of the inhibition zone around the well, and the determination of their minimal inhibitory concentration (MIC) and minimum bactericidal concentration. The activity tests were conducted using the diffusion disc and broth microdilution assays. Very weak antibacterial activity, with MIC values of $125.0 \mu \mathrm{g} / \mathrm{ml}$ against Staphylococcus aureus, Bacillus cereus, and Micrococcus luteus (MIC > $125.0 \mu \mathrm{g} / \mathrm{ml}$ ) was shown by this extract. No significant antifungal activity was observed [22]. 


\section{Conclusions}

The present paper summarizes the limited information on E. spinosus and highlights the therapeutic potential, which is used mainly as an anti-inflammatory drug in Algeria, as well as in Morocco. To the best of our knowledge, no study has been conducted to describe the toxicological effects of this species. Therefore, further clinical studies, based on standardized extracts from a sustainable source, must be designed to ensure the safety and efficacy of the extracts of this species which is widely used in traditional medicine in North Africa as an abortifacient drug.

\section{Acknowledgments}

The authors thank Professor Emeritus Dr. Geoffrey A. Cordell, Natural Products Inc. and University of Florida, Gainesville, FL, USA for his review of the manuscript prior to submission.

\section{References}

[1] Halim AF, Afify MS, Ahmed AF, Mira AS. The fact about Echinopsine and first isolation of echinorine from Echinops spinosus L. J Environ Sci 2011; 40:173-81.

[2] Global Compositae Checklist. 2009. https://compositae.landcareresearch.co.nz/?Page $=$ NameDetails\&NameId=C9849136-01E5-4A6A-9860-F575 3930D8D7 (Accessed 11 March 2018).

[3] The Plant List. 2013. Version 1.1 Published on the Internet, http://www.theplantlist.org/ (Accessed 11 March 2018).

[4] African Plant Database. Version 3.4.0. Conservatoire et Jardin botaniques de la Ville de Genève and South African National Biodiversity Institute, Pretoria. http://www.ville-ge.ch/musinfo/bd/cjb/africa/ details.php?langue $=$ fr\&id $=137404$ (Accessed 11 March 2018).

[5] IUCN. A guide of medicinal plants in North Africa. Centre for Mediterranean Cooperation, Malaga, Spain, pp 132-3, 2005.

[6] Quezel P, Santa S. Nouvelle flore de l'Algérie et des régions désertiques méridionale. Tome II. CNRS Editions, Paris, p 995, 1963.

[7] Ozenda P. Flore et végétation du Sahara. 3rd edition, CNRS Edition, Paris, p 446, 2004.

[8] Dobignard A, Chatelain C. Index synonymique de la flore d'Afrique du Nord. Volume 2, Editions des conservatoire et jardin botanique, Genève, p 258, 2011.

[9] Greuter W. The Euro+ Med treatment of Cardueae (Compositae) - generic concepts and required new names. Willdenowia 2003; 33:49-61.

[10] Abegaz BM, Tadesse M, Majinda R. Distribution of sesquiterepene lactones and polyacetylenic thiophenes in Echinops. Biochem System Ecol 1991; 19:323-28.

[11] Gheffour K, Boucherit K, Boucherit-Otmani Z. Etude phytochimique et évaluation de l'activité antioxydante des extraits d'Echinops spinosus. Rev Phytother 2015; 13(5):288-94.

[12] Boumaraf M, Benyahia S, Mekkiou R, Benayache S, Benayache F. Flavonoids from ethyl acetate extract of Echinops spinosus (Asteraceae). Der Pharma Chemica 2016; 8(8):158-60.

[13] Ait Youssef M. Plantes médicinales de Kabylie, Ibis Press, Paris, pp 117-9, 2006.

[14] Baba Aissa F. Encyclopédie des plantes utiles. Flore d'Algérie et du Maghreb, Librairie moderne, Rouiba, p 95, 2000.

[15] Rebahi A. Répertoire des noms des plantes du Maghreb (Arabe-Berbère-Latin Français). Avec des notes de leur utilisation dans le domaine alimentaire, fourrager et autres, Livres Editions, Alger, $\mathrm{p}$ 110, 2015.

[16] Sahki A, Boutamine Sahki R. Le Hoggar: promenade botanique. Éd. Ésope. Chamonix, Lyon, p 185, 2004.

[17] Bellakhdar J, Claisse R, Fleurentin J, Younos C. Repertory of standard herbal drugs in the Moroccan pharmacopoea. J Ethnopharmacol 1991; 35:123-43.

[18] Agyare C, Obiri DD, Boakye YD, Osafo N. Antiinflammatory and analgesic activities of African plants. In: (eds). Medicinal plant research in africa pharmacology and chemistry. 1st edition, Elsevier, London, pp 725-52, 2013.

[19] Chermat S, Gharzouli R. Ethnobotanical study of medicinal flora in the North-East of Algeria-an empirical knowledge in Djebel Zdimm (Setif). J Mater Sci Eng A 2015; 5(1-2):50-9.

[20] Hammiche V, Maiza K. Traditional medicine in Central Sahara: Pharmacopoeia of Tassili N'ajjer. J Ethnopharmacol 2006; 105:358-67.

[21] Hymetes A, Iversen TH, Rohloff J, Erko B. Screening of Echinops ellenbeckii and Echinops longisetus for biological activities and chemical constituents. Phytomedicine 2005; 12:675-9.

[22] Dong M, Cong B, Yu SH, Sauriol F, Huo CH, Shi QW, et al. Echinopines A and B: sesquiterpenoids possessing an unprecedented skeleton from Echinops spinosus. Org Lett 2008; 10(5):701-4.

[23] Ibrahim H, Moaty A. Chemical constituents of Echinops spinosissimus Turra. Int J Adv Res 2016; 4(7):1129-36.

[24] Bouattour E, Fakhfakh J, Dammak DF, Jabou K, Damak M, Mezghani Jarraya R. Hexane extract of Echinops spinosissimus Turra subsp. spinosus from Tunisia: a potential source of acetylated sterolsinvestigation of its biological activities. Chem Biodivers 2016; 13:1674-84.

[25] Wu Y, Li S, Zhang Z, Sauriol F. New thiophene acetylene from Echinops spinosissimus subsp. spinosus. Chem Nat Coump 2017; 53(5):933-4. 
[26] Ni ZW, Nagashima Y, Zhang ML, Wang YF, Dong M, Sauriol F, et al. 11-Hydroxyisocom-2-en-5-one, a new sesquiterpenoid from Echinops spinosissimus. Chem Nat Coump 2013; 49(4):632-4.

[27] Bouattour E, Fakhafakh J, Affes M, Chawech R, Damak M, Jarray R. Chemical constituents of Echinops spinosus from Tunisia. Chem Nat Coump 2017; 5:984-7.

[28] Chaudhuri P. Echinozolinone an alkaloid from Echinops echinatus. Phytochemistry 1992; 26(2):587-9.

[29] Pranav V, Vinay J, Priya M, Sudhir B, Neha A, Love C, et al. Echinops echinatus Roxb.-A nature's Drugstore: an overview. Indo Am J Pharmaceut Res 2013; 3(2):2744-50.

[30] Kiyekbayeva L, Mohamed NM, Yekebulan O, Mohamed EI, Ubaidilla D, Nursulu A, et al. Phytochemical constituents and antioxidant activity of Echinops albicaulis. Nat Prod Res 2017; 32(10):1203-7.

[31] Bhakuni RS, Shukla YN, Thakur RS. Alkaloides and lipid constituents of Echinops niveus. Phytochemistry 1990; 29(8):2697-8.

[32] Liu Y, Ye M, Guo HZ, Zhao YY, Guo DA. New thiophenes from Echinops grijisii. J Asian Nat Prod Res 2002; 4(3):175-8.

[33] Singh RP, Pandey VB. Further flavonoids of Echinops niveus. Fitoterapia 1994; 65(4):374.

[34] Ibrahim SRM, Abdallah HM, El-Halawany AM, Mohamed GA. Naturally occurring thiophenes: isolation, purification, structural elucidation and evaluation of bioactivities. Phytochem Rev 2016; 15:197-220.

[35] Kheder O, Moussaoui Y, Bensalem R. Solvent effects on phenolic contents and antioxidant activities of the Echinops spinosus and the Limoniastrum monopeltatum. Research Journal of Pharmaceutical, Biological and Chemical Sciences 2014; 5(2):66-76.

[36] Parhat R, Makabel B, Nurhabek U, Tohonrbek A, Hayni, Song FF, et al. Overview of application and research of Echinops genus in Chinese medicine. Zhongguo Zhong Yao Za Zhi 2014; 39(19):3865-9.

[37] Boulos L. Medicinal plants of North Africa. Reference Publications Inc., Algonac, MI, p 286, 1983.

[38] Bnouham M, Mekhfi H, Legssyer A. Medicinal plants used in the treatment of diabetes in Morocco. Int J Diabet Metab 2002; 10:33-50.

[39] Hseini S, Kahouadji A. Etude ethnobotanique de la flore médicinale dans la région de Rabat (Maroc occidental). Lazaroa 2007; 28:79-93.

[40] Lahsissene H, Kahouadjia A, Tijane M, Hseini S. Catalogue des plantes médicinales utilisées dans la région de Zaer (Maroc occidental). Lejeunia 2009; 186:1-25.

[41] Abouri M, El Mousadik A, Msanda F, Boubaker H, Saadi B, Cherif K. An ethnobotanical survey of medicinal plants used in the Tata Province, Morocco. Int J Med Plants Res 2012; 1(7):99-123.
[42] Alami Z, Aynaou H, Alami B, Hdidou Y, Latrech H. Herbal medicines use among diabetic patients in Oriental Morocco. J Pharmacognosy Phytother 2015; 7(2):9-17.

[43] Sbai-Jouilil H, Fadli A, Zidane L. Survey of ethnomedicinal plants used for the treatment of gastrointestinal disorders in Seksaoua region (Western High Moroccan Atlas). Annu Res Rev Biol 2017; 16(5):1-9.

[44] Baghdad M, Boumediene M, Djamel A, Djilali B. Ethnobotany of medicinal plants in the region Béni chougrane (Mascara, Algeria). J Bio Environ Sci 2016; 9(1):426-33.

[45] Miara MD, Ait Hammou M, Hadjadj Aoul S. Phytothérapie et taxonomie des plantes médicinales spontanées dans la région de Tiaret (Algérie). Rev Phytother 2013; 11:206-18.

[46] Talhouk RS, Karam C, Fostok S, El-Jouni W, Barbour EK. Antiinflammatory bioactivities in plant extracts. J Med Food 2007; 10(1):1-10.

[47] Rimbau V, Cerdan C, Vila R, Iglesias J. Antiinflammatory activity of some extracts from plants used in the traditional medicine of North-Africa countries (II). Phytother Res 1999; 13:128-32.

[48] Winter CA, Risley EA, Nuss GW. Carrageenininduced oedema in the hind paw of the rat as an assay for anti-inflammatory drugs. Proc Soc Exp Biol Med 1962; 111:544-7.

[49] Rimbau V, Risco E, Canigueral SR, Iglesias J. Antiinflammatory activity of some extracts from plants used in the traditional medicine of NorthAfrica countries. Phytother Res 1996; 10:421-3.

[50] Young JM, Spires SA, Bedord CJ, Wagner B, Ballaron SJ, De Young LM. The mouse ear inflammatory response to topical arachidonic acid. J Invest Dermatol 1984; 82:367-71.

[51] Singh B, Gambhir SS. Anti-inflammatory activity of Echinops echinatus. J Ethnopharmacol 1989; 25:189-99.

[52] Singh B, Ram SN, Pandey VB, Joshi VK, Gambhir SS. Studies on anti-inflammatory activities of taraxasterol acetate from Echinops echinatus in rats and mice. Phytother Res 1991; 5:103-6.

[53] Yadava RN, Singh SK. New anti-inflammatory active flavanone glycoside from the Echinops echinatus Roxb. Indian J Chem 2006; 45B:1004-8.

[54] Erenler R, Yilmaz S, Aksit H, Sen O, Nusret G, Elmastas M, et al. Antioxidant activities and chemical constituents isolated from Echinops orientalis Trauv. Rec Nat Prod 2014; 8(1):32-6.

[55] Aydin C, Ozcan GT, Turan M, Mammadov R. Phenolic contents and antioxidant properties of Echinops ritro L. and E. tournefortii Jaup. et Spach extract. Int J Sec Metabolite 2016; 3(2):74-81. 